\title{
General Prediction of Peptide-MHC Binding Modes Using Incremental Docking: A Proof of Concept
}

\author{
Dinler A. Antunes \\ Department of Computer Science \\ Rice University \\ Houston, Texas, USA \\ dinler@rice.edu
}

\author{
Didier Devaurs \\ Department of Computer Science \\ Rice University \\ Houston, Texas, USA \\ devaurs@rice.edu
}

\author{
Mark Moll \\ Department of Computer Science \\ Rice University \\ Houston, Texas, USA \\ mmoll@rice.edu
}

\author{
Gregory Lizée \\ Department of Melanoma Medical \\ Oncology - Research, The University \\ of Texas MD Anderson Cancer Center \\ Houston, Texas, USA \\ glizee@mdanderson.org
}

\author{
Lydia E. Kavraki* \\ Department of Computer Science \\ Rice University \\ Houston, Texas, USA \\ kavraki@rice.edu
}

\begin{abstract}
The class I major histocompatibility complex (MHC) is capable of binding peptides derived from intracellular proteins and displaying them at the cell surface. The recognition of these peptide-MHC (pMHC) complexes by T-cells is the cornerstone of cellular immunity, enabling the elimination of infected or tumoral cells. Tcell-based immunotherapies against cancer, which leverage this mechanism, can greatly benefit from structural analyses of pMHC complexes. Several attempts have been made to use molecular docking for such analyses, but pMHC structure remains too challenging for even state-of-the-art docking tools. To overcome these limitations, we describe the use of an incremental meta-docking approach for structural prediction of pMHC complexes. Previous methods applied in this context used specific constraints to reduce the complexity of this prediction problem, at the expense of generality. Our strategy makes no assumption and can potentially be used to predict binding modes for any pMHC complex. Our method has been tested in a re-docking experiment, reproducing the binding modes of 25 pMHC complexes whose crystal structures are available. This study is a proof of concept that incremental docking strategies can lead to general geometry prediction of $\mathrm{pMHC}$ complexes, with potential applications for immunotherapy against cancer or infectious diseases.
\end{abstract}

\section{CCS CONCEPTS}

- Computing methodologies $\rightarrow$ Molecular simulation; • Applied computing $\rightarrow$ Molecular structural biology;

\footnotetext{
${ }^{*}$ Corresponding author.
}

Permission to make digital or hard copies of part or all of this work for personal or classroom use is granted without fee provided that copies are not made or distributed for profit or commercial advantage and that copies bear this notice and the full citation on the first page. Copyrights for third-party components of this work must be honored For all other uses, contact the owner/author(s).

ACM-BCB'18, August 29-September 1, 2018, Washington, DC, USA

(C) 2018 Copyright held by the owner/author(s).

ACM ISBN 978-1-4503-5794-4/18/08.

https://doi.org/10.1145/3233547.3233719

\section{KEYWORDS}

major histocompatibility complex (MHC), peptide-MHC complex, molecular docking, geometry prediction, cancer immunotherapy

\section{ACKNOWLEDGEMENTS}

This work was partially supported by NIH (grant number 1R21CA20994101), through the Informatics Technology for Cancer Research (ITCR) initiative of the National Cancer Institute (NCI), and by a training fellowship from the Gulf Coast Consortia, on the Computational Biology Training Program (CPRIT Grant No. RP170593). Additionally, this work was partially supported by a fellowship (202186/20148) from CNPq (Conselho Nacional de Desenvolvimento Cient Ãyfico $e$ Tecnol Âşgico), under the Brazilian Scientific Mobility Program. Finally, this work was also partially supported by the Big-Data Private-Cloud Research Cyberinfrastructure MRI-award funded by NSF under grant CNS-1338099 and by Rice University.

\section{ADDITIONAL INFORMATION}

This work has been published as an open-access article in [1]. Our incremental docking tool, DINC, is freely available as a web-server at http://dinc.kavrakilab.org, along with technical documentation and video tutorials.

\section{REFERENCES}

[1] Dinler A. Antunes, Didier Devaurs, Mark Moll, Gregory Lizée, and Lydia E. Kavraki. 2018. General prediction of peptide-MHC binding modes using incremental docking: A proof of concept. Scientific Reports 8 (2018), 4327. https://doi.org/10. 1038/s41598-018-22173-4 